\section{Mitchell, Scott, and Mitchell are not supported by their own data}

\author{
JOHN GARCIA \\ Departments of Psychology and Psychiatry \\ University of California at Los Angeles \\ Los Angeles, California 90024
}

Rather than reply to Mitchell's reply to Revusky, I suggest we all examine the data in the paper at issue (Mitchell, Scott, \& Mitchell, 1977). These data do not support Mitchell's hypothesis; instead, they tend to support the alternative flavor-illness association hypothesis which he is disputing. The authors make the Mitchell hypothesis clear enough. They entitled their paper, "Attenuated and enhanced neophobia in the taste-aversion 'delay of reinforcement' effect " and suggest in their abstract that "taste aversion obtained when laboratory rats are poisoned some hours after the consumption of a novel substance consists of two antagonistic nonassociative components, attenuation or habituation of neophobia and disruption of habituation by the administration of a noxious stimulus during the habituation process."

First, let us consider their methods. Throughout the experiment, all rats are deprived of water except for a 30-min test period each day. On the single acquisition day, six experimental groups are formed. Animals drink either water or saccharin-flavored water from a single bottle, after which they are injected with saline immediately, toxin immediately, or toxin after a 6-h delay. Beginning the next day, all groups are presented simultaneously with water and saccharin-flavored water in a two-bottle test for 10 consecutive days.

Now consider the water groups. They encounter saccharin for the first time in the two-bottle test after acquisition. All water groups display neophobia, initially drinking little saccharin-flavored water and then habituate rapidly. The same steep habituation slope is displayed by all water groups regardless of whether they received saline, toxin, or delayed toxin injections. As the authors themselves point out, there is no evidence of enhanced neophobia or disruption of the habituation process; therefore, the Mitchell hypothesis is not supported.

Finally, consider the saccharin groups. Toxic injections produce an orderly delay-of-reinforcement gradient. Immediate toxic injection produces a strong aversion and a shallow extinction slope. Delayed injections produce a lesser aversion but they appear to be clearly different from the saline controls until their orderly extinction reaches the saline controls on about Day 7. Therefore, the flavor- illness association hypothesis is supported, although admittedly there is better evidence in the literature.

Neophobia and conditioned aversions are both real phenomena and they both initially depress fluid consumption, but in this study they exhibit different slopes in Figure 2. Mitchell's echo of Bitterman's (1976) criticism that we failed to employ nonassociative controls is incomprehensible, since Mitchell et al. used the same general design as Garcia and Kimeldorf (1960) and Garcia, Kimeldorf, and Koelling (1955). We also used six groups drinking water or saccharin in conjunction with two levels of radiation and sham exposure. We measured preferences before and after exposure, and found no evidence for disruption of neophobia habituation or pseudoconditioning. Others employing similar controls demonstrated that exposure to radiation produces delayed noxious reinforcement (e.g., Smith, Taylor, Morris, \& Hendricks, 1965).

As the authors candidly admit, "The present results do not prove that rats poisoned some hours after consuming a novel saccharin solution do not learn a saccharin-illness aversion." Furthermore, they frankly admit that they failed to support their own thesis, perhaps due to errors or bad luck, concluding that: "Failure to demonstrate a significant sensitization effect in the present experiment may be due to the formation of a weak conditioned avoidance of water (Group P-W-I), to a 'floor effect' imposed by the use of a two-bottle test, or to fortuitous group differences in initial neophobia or saccharin preferences." (Note: P-W-I group is not different from any other water group.) It is difficult to be critical in the face of such candor, especially when they apparently published this paper to help us avoid procedural errors and inappropriate inferences in our research.

\section{REFERENCES}

Brtterman, M. E. Flavor aversion studies. Science, 1976, 192. 265-267.

GARCIA, J., \& KIMELDORF, D. J. Conditioned avoidance behavior induced by low-dose neutron exposure. Nature, 1960, 185, 261-262.

Garcia, J., Kimgldorf, D. J., \& Koelling, R. A. Conditioned aversion to saccharin resulting from exposure to gamma radiation. Science, $1955,122,157-158$.

Mrtchell, D., Scott, D. W., \& Mrtchell, L. K. Attenuated and enhanced neophobia in the taste-aversion "delay of reinforcement" effect. Animal Learning \& Behavior, 1977, 5, 99-102.

Smith, J. C., TaYLoR, H. L., Morris, D. D., \& Hendricks, J. Further studies of $\mathrm{x}$-ray conditioned saccharin aversion during the postexposure period. Radiation Research, 1965, 24, 423-431.

(Received for publication May 17, 1977; accepted October 20, 1977.) 